\title{
LE COMPLÉMENTAIRE DES PUISSANCES $n$-IÈMES DANS UN CORPS DE NOMBRES EST UN ENSEMBLE DIOPHANTIEN
}

\author{
par \\ Jean-Louis Colliot-Thélène \& Jan Van Geel
}

Résumé. - Le cas $n=2$ de l'énoncé du titre est un théorème de $\mathrm{B}$. Poonen (2009), qui utilise une famille à un paramètre de variétés et un théorème sur l'obstruction de Brauer-Manin pour les points rationnels de ces variétés dû à Coray, Sansuc et l'un des auteurs (1980). Pour $n=p$ premier quelconque, on dispose d'une famille de variétés analogues, qui ont été considérées par A. Várilly-Alvarado et B. Viray (2012). Nous obtenons le résultat annoncé en remplaçant ladite famille par sa puissance symétrique $(2 p+1)$-ième, et en appliquant un théorème sur l'obstruction de Brauer-Manin pour les points rationnels de telles puissances symétriques reposant sur des travaux de l'un des auteurs avec Swinnerton-Dyer (1994) et avec Skorobogatov et SwinnertonDyer (1998), qui généralisent un travail de Salberger (1988).

Samenvatting. - Voor $n=2$ is de bewering in de titel een stelling van B. Poonen (2009). Hij gebruikt een één-parameter familie van variëteiten, en een stelling van Coray, Sansuc en één van de auteurs (1980), over de BrauerManin obstructie voor de rationale punten van deze variëteiten. Voor $n=p$, $p$ een willekeurig priemgetal, beschouwden A. Várilly-Alvarado en B. Viray (2012) een analoge familie van variëteiten. We bewijzen de bewering in de titel door deze familie te vervangen door de $(2 p+1)$-de symmetrische macht ervan en door een stelling over de Brauer-Manin obstructie voor de rationale punten van zulke symmetrische machten toe te passen. Deze stelling steunt op werk van één van de auteurs met Swinnerton-Dyer (1994) en met Skorobogatov en Swinnerton-Dyer (1998). Dat werk veralgemeent resultaten van Salberger (1988) 


\section{Introduction}

Soient $k$ un corps et $n>0$ un entier. Un sous-ensemble $D$ de $\mathbf{A}^{n}(k)=k^{n}$ est dit diophantien s'il existe une $k$-variété $Z$ et un $k$-morphisme $f: Z \rightarrow$ $\mathbf{A}_{k}^{n}$ tel que $D=f(X(k))$. Dans cette définition, on peut supposer $Z$ affine. On peut même supposer que $Z$ est un sous-schéma fermé de $\mathbf{A}_{k}^{m+n}$ pour un certain $m$ et que le morphisme $f$ est induit par la projection $\mathbf{A}_{k}^{m+n} \rightarrow \mathbf{A}_{k}^{n}$. Une union finie de sous-ensembles diophantiens dans $\mathbf{A}^{n}(k)$ est un sous-ensemble diophantien. Nous montrons (Théorème 4.3) :

Pour tout corps de nombres $k$ et tout entier $r>1$, le complémentaire dans $k^{\times}$de l'ensemble $k^{\times r}$ des puissances $r$-ièmes est un ensemble diophantien.

Pour $r$ une puissance de 2, c'est un théorème de B. Poonen [10]. Une démonstration "élémentaire" du cas $r=2$ sur $k=\mathbb{Q}$ vient d'être donnée par J. Königsmann [9, Prop. 20 (b)].

Dans le cas $r=2$, l'argument de Poonen utilise les surfaces de Châtelet d'équation affine

$$
y^{2}-a z^{2}=P(x)
$$

avec $a \in k^{\times}$et $P(x)$ polynôme séparable produit de deux polynômes $Q(x)$ et $R(x)$ de degré 2 . Pour de telles surfaces, il est établi par Colliot-Thélène, Coray et Sansuc [3] que l'obstruction de Brauer-Manin est la seule obstruction au principe de Hasse pour les points rationnels sur de telles surfaces. Ce résultat est généralisé dans [4] à tout polynôme $P(x)$ de degré 4 .

Poonen [10] part d'un contre-exemple au principe de Hasse d'équation $y^{2}-b z^{2}=Q(x) R(x)$ (il en existe [11]), puis considère la famille $\mathbb{U}_{u}$ à un paramètre $u \in k^{\times}$de surfaces $U_{u}$ d'équation $y^{2}-b u z^{2}=Q(x) R(x)$.

Un point clé est que l'ensemble des $u \in k^{\times}$sans obstruction de BrauerManin pour l'existence de points rationnels sur $\mathbb{U}_{u}$ est un ensemble diophantien, car d'après [3], c'est l'image des points $k$-rationnels de $\mathbb{U}_{u}$ par le $k$ morphisme $\mathbb{U}_{u} \rightarrow \mathbb{G}_{m, k}$ donné par la coordonnée $u$.

Dans le cas $r=p$ avec $p$ premier quelconque, on considère les variétés définies sur un corps de nombres $k$ contenant une racine $p$-ième de 1 par une équation

$$
\operatorname{Norm}_{k\left((b u)^{1 / p}\right) / k}(\Xi)=P(x)
$$

avec $b \in k^{\times}$fixe, $u$ variant dans $k^{\times}$, et $P(x)$ un polynôme séparable produit de deux polynômes $Q(x)$ et $R(x)$ de degré $p$. Sous l'hypothèse de Bouniakowsky-Dickson-Schinzel, Colliot-Thélène et Swinnerton-Dyer [5] montrent que l'obstruction de Brauer-Manin est la seule obstruction au principe de Hasse pour les points rationnels de telles variétés. C'est ce 
qui a permis à A. Várilly-Alvarado et B. Viray [15] d'étendre, de façon conditionnelle, l'argument de Poonen à tout entier $r$.

L'article [5] établit aussi un résultat inconditionnel : pour des variétés du type ci-dessus, l'obstruction de Brauer-Manin à l'existence d'un zéro-cycle de degré 1 est la seule obstruction [5, Thm. 5.1]. C'est une généralisation d'un résultat de Salberger [13] sur les surfaces fibrées en coniques.

L'idée nouvelle du présent article est d'utiliser une version « effective $\gg$ de ce résultat (Corollaire 3.2) : Il existe un entier $N$, premier à $p$, indépendant de $u$, avec la propriété suivante. Pour $u \in k^{\times}$, sur un modèle projectif et lisse convenable $\mathbb{X}_{u}$ d'une variété ci-dessus, s'il n'y a pas d'obstruction de BrauerManin à l'existence d'un point rationnel, alors il existe un zéro-cycle effectif de degré $N$ sur $\mathbb{X}_{u}$, ce qui se traduit par le fait que le produit symétrique $\operatorname{Sym}^{N} \mathbb{X}_{u}$ possède un $k$-point. Cela permet de montrer que le complémentaire de l'ensemble des points $u \in k^{\times}$pour lesquels $\mathbb{X}_{u}\left(\mathbf{A}_{k}\right) \neq \emptyset$ et $\mathbb{X}_{u}\left(\mathbf{A}_{k}\right)^{\mathrm{Br}}=\emptyset$ est un ensemble diophantien. Le reste de l'argument (Théorème 4.3) est alors comme dans [10].

Pour établir le résultat d'effectivité, nous reprenons les démonstrations de [5], dans la version plus souple développée dans [6] (Théorème 3.1 et Corollaire 3.2). La méthode donne $N=2 p+1$. Comme nous l'a signalé O. Wittenberg au vu d'une précédente version de cet article, un résultat d'effectivité plus général a déjà été obtenu, par la même méthode, dans son article [17].

Si l'on admet un résultat algébrique annoncé par Salberger dans sa thèse [12], mais non publié, on peut se dispenser de revisiter les démonstrations des articles [5] ou [6], ou de citer [17]. Cette méthode alternative donne $N=(p-1)^{2}$. Le résultat de Salberger [12] est décrit au $\S 6$, la variante de la démonstration du théorème principal étant exposée au $\S 5$.

\section{Rappels et notations}

On note $A[n]$ le sous-groupe de $n$-torsion d'un groupe abélien $A$.

Soient $k$ un corps et $n$ un entier. On appelle extension cyclique $K$ de $k$ de degré $n$ une $k$-algèbre étale $K$ et une action de $G=\mathbb{Z} / n$ sur $K$ qui fait de Spec $K \rightarrow$ Spec $k$ un $G$-torseur. En particulier par extension cyclique de corps $K / k$, on entend dans ce texte une extension cyclique galoisienne de groupe $G=\mathbb{Z} / n$ muni du générateur $1 \in \mathbb{Z} / n$. Ceci définit une classe $\chi_{K / k} \in H^{1}(k, \mathbb{Z} / n)$. Si $n \neq 0 \in k$, à tout élément $c \in k^{\times}$on associe sa classe dans $k^{\times} / k^{\times n} \stackrel{\widetilde{\sim}}{\rightarrow} H^{1}\left(k, \mu_{n}\right)$.

Pour $K / k$ une extension cyclique de degré $n$ premier à la caractéristique de $k$, et $c \in k^{\times}$, on dispose de l'algèbre simple centrale cyclique $(K / k, c)$ de 
degré $n$, dont on note encore $(K / k, c)$ la classe dans le groupe de Brauer Brk, qui est définie comme le cup-produit via

$$
H^{1}(k, \mathbb{Z} / n) \times H^{1}\left(k, \mu_{n}\right) \rightarrow H^{2}\left(k, \mu_{n}\right)
$$

de la classe $\chi_{K / k}$ et de la classe de $c$ dans $k^{\times} / k^{\times n}$.

Supposons que $k$ contient une racine primitive $n$-ième de 1 , soit $\zeta$. Le choix d'un isomorphisme $\mathbb{Z} / n \stackrel{\simeq}{\rightarrow} \mu_{n}$ permet d'identifier $H^{1}(k, \mathbb{Z} / n)=$ $H^{1}\left(k, \mu_{n}\right)=k^{\times} / k^{\times n}$ et $\left.(\operatorname{Br} k)\right][n]=H^{2}\left(k, \mu_{n}\right)=H^{2}\left(k, \mu_{n}^{\otimes 2}\right)$. Étant donnés $b, c \in k^{\times}$, on note alors $(b, c)_{\zeta} \in(\operatorname{Br} k)[n]$ le cup-produit des classes $b$ et $c$ dans $k^{\times} / k^{\times n}=H^{1}\left(k, \mu_{n}\right)$. Compte tenu de l'identification $\mathbb{Z} / n=\mu_{n}$, la $k$-algèbre $k\left(b^{1 / n}\right):=k[t] /\left(t^{n}-b\right)$ est munie naturellement d'une structure d'extension cyclique de degré $n$, et l'on a $\left(k\left(b^{1 / n}\right) / k, c\right)=(b, c)_{\zeta} \in \operatorname{Br} k$.

La lecture du présent article requiert une certaine familiarité avec les articles $[\mathbf{5}],[\mathbf{6}],[\mathbf{1 0}]$.

\section{Algèbre}

Le lemme suivant est bien connu (cf. [8, Chapitres 4 et 5]).

Lemme 2.1. - Soit $K / k$ une extension cyclique de corps de degré n premier à la caractéristique de $k$. Soit $c \in k^{\times}$.

(i) La k-variété affine $Y$ d'équation $\operatorname{Norm}_{K / k}(\Xi)=c$ est un ouvert de la $k$-variété de Severi-Brauer $X$ d'indice $n-1$ attachée à l'algèbre simple centrale $(K / k, c)$.

(ii) La k-variété $Y$ possède un k-point si et seulement si la classe de $(K / k, c)$ est nulle dans Brk.

(iii) On a une suite exacte

$$
\mathbb{Z} / n \rightarrow \operatorname{Br} k \rightarrow \operatorname{Br} X \rightarrow 0,
$$

où $1 \in \mathbb{Z} / n$ a pour image $(K / k, c) \in \operatorname{Br} k$.

Démonstration. - En utilisant $[\mathbf{8}]$, on établit ce lemme bien connu, à un point près. L'exercice [8, Ex. 1], où il convient de remplacer $b x$ par $b x^{n}$, donne un énoncé d'équivalence birationnelle stable au lieu de (i) ci-dessus. Esquissons comment l'on obtient (i). On a les suites exactes de $k$-tores

$$
1 \rightarrow R_{K / k}^{1} \mathbb{G}_{m} \rightarrow R_{K / k} \mathbb{G}_{m} \stackrel{\text { Norm }_{K / k}}{\longrightarrow} \mathbb{G}_{m, k} \rightarrow 1
$$

et

$$
1 \rightarrow \mathbb{G}_{m, k} \stackrel{x \mapsto x}{\longrightarrow} R_{K / k} \mathbb{G}_{m} \rightarrow R_{K / k} \mathbb{G}_{m} / \mathbb{G}_{m, k} \rightarrow 1 .
$$


Notons $T_{1}=R_{K / k}^{1} \mathbb{G}_{m}$ et $T=R_{K / k} \mathbb{G}_{m} / \mathbb{G}_{m, k}$. Le choix d'un générateur de $\operatorname{Gal}(K / k)$ définit un isomorphisme de $k$-tores $T_{1} \stackrel{\sim}{\rightarrow} T$. La $k$-variété $Y$ est un espace principal homogène sous le $k$-tore $T_{1}$. C'est donc aussi un espace principal homogène sous le $k$-tore $T$. On a un plongement torique, de $R_{K / k} \mathbb{G}_{m}$ dans $W:=R_{K / k} \mathbb{G}_{a} \backslash\{0\} \simeq \mathbf{A}_{k}^{n} \backslash\{0\}$, qui induit un plongement torique de $T$ dans le quotient de $W$ par l'action diagonale de $\mathbb{G}_{m, k}$, quotient qui s'identifie à l'espace projectif $\mathbf{P}_{k}^{n-1}$. La $k$-variété $Y$ est donc un ouvert dans la $k$-variété $X=Y \times^{T} \mathbf{P}_{k}^{n-1}$ quotient de $Y \times \mathbf{P}_{k}^{n-1}$ par l'action diagonale de $T$. Cette $k$-variété $X$ est une forme tordue de l'espace projectif. On a le diagramme commutatif de $k$-groupes

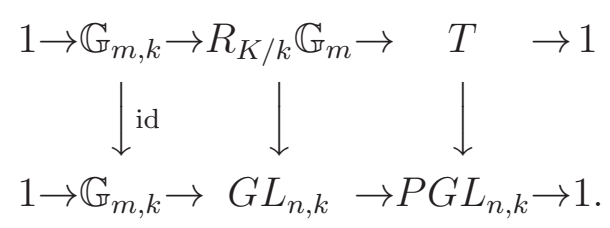

Ce diagramme induit un diagramme commutatif

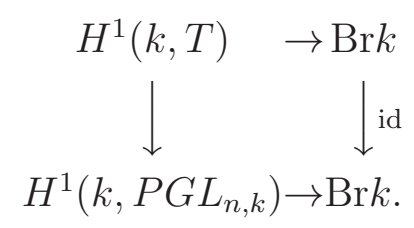

La flèche $H^{1}(k, T) \rightarrow H^{1}\left(k, P G L_{n, k}\right)$ envoie la classe de $Y$ sur la classe de $X$, et le composé $k^{\times} / N_{K / k} K^{\times} \stackrel{\simeq}{\rightarrow} H^{1}\left(k, T_{1}\right) \stackrel{\simeq}{\rightarrow} H^{1}(k, T) \rightarrow$ Brk envoie la classe de $c$ sur la classe de l'algèbre cyclique $(K / k, c)$ (cf. [8, Cor. 4.7.4]). Quant à la flèche $H^{1}\left(k, P G L_{n, k}\right) \rightarrow$ Brk, elle envoie la classe d'isomorphie d'une variété de Severi-Brauer d'indice $n-1$ sur sa classe dans le groupe de Brauer.

Proposition 2.2. - Soit $k$ un corps de caractéristique zéro. Soient $p$ un nombre premier et $P(x) \in k[x]$ un polynôme séparable de degré $2 p$. Soit $K / k$ une extension galoisienne cyclique, de groupe $G=\mathbb{Z} / p$. Soit $U$ la $k$-variété définie par

$$
\operatorname{Norm}_{K / k}(\Xi)=P(x) \neq 0 .
$$

(i) Il existe une $k$-compactification lisse $X$ de $U$, équipée d'un morphisme $\pi: X \rightarrow \mathbf{P}_{k}^{1}$ étendant l'application $(\Xi, x) \mapsto x$, dont la fibre générique $X_{\eta} / k\left(\mathbf{P}^{1}\right)$ est une variété de Severi-Brauer de dimension $p-1$, d'algèbre simple centrale associée l'algèbre cyclique $(K / k, P(x))$, telle que pour $M$ 
point fermé de $\mathbf{P}_{k}^{1}$ non zéro de $P(x)$, la fibre $X_{M}$ est une variété de SeveriBrauer sur le corps résiduel $k(M)$.

(ii) Si K n'est pas un corps, alors la k-variété $X$ est $k$-birationnelle à $\mathbf{P}_{k}^{p}$, et $\operatorname{Br} k=\operatorname{Br} X$.

(iii) Supposons que $K$ est un corps, et que $P(x)=Q(x) \cdot R(x)$ avec $Q(x)$ et $R(x)$ irréductibles de degré $p$ et sans zéro dans $K$. Alors les algèbres cycliques $(K / k, Q(x))$ et $(K / k, R(x))$ dans $\operatorname{Br} k(x)$ ont une image dans $\mathrm{Br} k(X)$ qui appartient à $\mathrm{Br} X$, et le quotient de $\mathrm{Br} X$ par l'image de $\mathrm{Brk}$ est un groupe cyclique d'ordre p engendré par l'image de $(K / k, Q(x))$.

Démonstration. - (1) On considère la $k$-variété d'équation

$$
\operatorname{Norm}_{K / k}(\Xi)=P(x)
$$

et $U_{1} \subset U$ l'ouvert maximal sur lequel la projection $U_{1} \rightarrow \mathbf{A}_{k}^{1}=\operatorname{Spec} k[x]$ définie par $x$ est lisse.

Soit $V$ la $k$-variété $V$ d'équation

$$
\operatorname{Norm}_{K / k}(\Theta)=P^{\prime}(y)
$$

avec $P^{\prime}(y):=y^{2 p} P(1 / y)$. Soit $V_{1} \subset V$ l'ouvert maximal sur lequel le morphisme $V_{1} \rightarrow \mathbf{A}_{k}^{1}=$ Spec $k[y]$ défini par $y$ est lisse.

On recolle la $k$-variété $U_{1}$ et la $k$-variété $V_{1}$ en une $k$-variété $W$ via $x=1 / y$ et $y^{2} . \Xi=\Theta$. On dispose donc d'un $k$-morphisme $W \rightarrow \mathbf{P}_{k}^{1}$ à fibres lisses, dont toutes les fibres sauf celles au-dessus des points fermés à support dans $P(x)=0$ sont géométriquement intègres.

Une variante du lemme 2.1 permet alors de construire une $k$-variété $W^{\prime}$ lisse équipée d'un morphisme $W^{\prime} \rightarrow \mathbf{P}_{k}^{1}$ dont les fibres au-dessus des points fermés autres que ceux supportés dans $P(x)=0$ sont des variétés de SeveriBrauer, et dont les fibres en les points fermés supportés dans $P(x)=0$ sont les fibres de $W \rightarrow \mathbf{P}_{k}^{1}$. En utilisant le théorème d'Hironaka, on obtient alors l'existence d'une $k$-variété projective $X$ munie d'un k-morphisme $\pi: X \rightarrow$ $\mathbf{P}_{k}^{1}$, contenant $W^{\prime}$ comme ouvert, le morphisme $\pi$ étendant $W^{\prime} \rightarrow \mathbf{P}_{k}^{1}$.

L'énoncé (ii) est évident : si $K$ n'est pas un corps, alors $\operatorname{Norm}_{K / k}(\Xi)$ s'écrit comme un produit de variables indépendantes, et la $k$-variété définie par l'équation

$$
Y_{1} \ldots Y_{p}=P(x) \neq 0
$$

est clairement $k$-rationnelle.

1. La proposition [15, Prop. 2.1] est incorrecte; nous ne pouvons donc citer [15, §3]. 
L'énoncé (iii) est essentiellement démontré dans [5, §2.2, Thm. 2.2.1]. Nous donnons les points principaux de la démonstration, renvoyant le lecteur à [5, §1 et $\S 2]$ pour des explications plus détaillées.

On fixe un générateur $\sigma$ de $\operatorname{Gal}(K / k)$, et on note $(K / k, c)=(K / k, \sigma, c)$.

On a la suite exacte (Lemme 2.1)

$$
\mathbb{Z} / p \cdot(\alpha) \rightarrow \operatorname{Brk}\left(\mathbf{P}^{1}\right) \stackrel{\pi^{*}}{\longrightarrow} \operatorname{Br} X_{\eta} \rightarrow 0,
$$

où $\alpha=(K(x) / k(x), P(x))$, qu'on note $(K / k, P(x))$. Soit $\gamma \in \operatorname{Br} X \subset$ $\operatorname{Br} X_{\eta}$. Il existe donc $\beta \in \operatorname{Brk}\left(\mathbf{P}^{1}\right)$ tel que $\gamma=\pi^{*}(\beta)$.

Les fibres de $\pi$ aux points fermés de $\mathbf{A}_{k}^{1}=$ Spec $k[x]$ autres que ceux définis par $Q=0$ et $R=0$ sont lisses et géométriquement intègres. La comparaison des résidus de $\gamma$ et de $\beta$ entraîne que les résidus de $\beta$ en tout point fermé de $\mathbf{A}_{k}^{1}$ autre que $Q=0$ et $R=0$ sont nuls. La comparaison des résidus de $\gamma$ et de $\beta$ au-dessus de $Q=0$, resp. de $R=0$, sur l'ouvert $U_{1} \subset X$, montre aussi que $\operatorname{Res}_{Q}(\beta) \in H^{1}\left(k_{Q}, \mathbb{Q} / \mathbb{Z}\right)$, resp. $\operatorname{Res}_{R}(\beta) \in$ $H^{1}\left(k_{R}, \mathbb{Q} / \mathbb{Z}\right)$, où $k_{Q}$, resp. $k_{R}$, désigne le corps résiduel en $Q=0$, resp. en $R=0$, s'annulent par passage à $K \otimes_{k} k_{Q}$, resp. $K \otimes_{k} k_{R}$. Les groupes $H^{1}\left(K \otimes_{k} k_{Q} / k_{Q}, \mathbb{Q} / \mathbb{Z}\right) \simeq \mathbb{Z} / p$, et $H^{1}\left(K \otimes_{k} k_{R} / k_{R}, \mathbb{Q} / \mathbb{Z}\right) \simeq \mathbb{Z} / p$ sont engendrés par l'image d'un générateur $\chi$ de $H^{1}(K / k, \mathbb{Q} / \mathbb{Z}) \simeq \mathbb{Z} / p$. La $k$ algèbre $(K / k, Q(x))$ a sur $\mathbf{A}_{k}^{1}$ pour seul résidu $\chi_{k_{Q}}$ en $Q=0$. La $k$-algèbre $(K / k, R(x))$ a sur $\mathbf{A}_{k}^{1}$ pour seul résidu $\chi_{k_{R}}$ en $R=0$. On voit donc qu'il existe des entiers $r$ et $s$ tels que $\beta-\left(K / k, Q(x)^{r}\right)-\left(K / k, R(x)^{s}\right)$ ait tous ses résidus triviaux sur $\mathbf{A}_{k}^{1}$, donc par la suite exacte de Faddeev (cf. [5, §1.2]) soit dans l'image de $\operatorname{Br} k$. Comme la classe $\alpha=(K / k, P(x)) \in \operatorname{Br} k\left(\mathbf{P}^{1}\right)$ a une image nulle dans $\operatorname{Br} k(X)$, on voit que $\gamma \in \operatorname{Br} X \subset \operatorname{Br} k(X)$ est la somme d'un multiple de $\pi^{*}(K / k, Q(x))$ et d'une classe dans Br $k$. Nous renvoyons à [5, Thm. 2.2.1] pour la démonstration du fait que $\pi^{*}(K / k, Q(x))$ appartient à $\operatorname{Br} X$, démonstration qui utilise le fait que le degré de $Q$ est $p$. Si la classe $\pi^{*}(K / k, Q(x)) \in \operatorname{Br} k(X)$ appartenait à l'image de $\operatorname{Br} k$, d'après le lemme2.1 il existerait un entier $r$ tel que

$$
(K / k, Q(x))-r(K / k, P(x))=(K / k, Q(x))-r(K / k, Q(x) R(x)) \in \operatorname{Br} k(x)
$$

appartienne à $\mathrm{Br} k$. Le calcul du résidu d'un tel élément au point fermé $R(x)=$ 0 donne $r=0$. Et le résidu de $(K / k, Q(x))$ en $Q(x)=0$ n'est pas nul. Ainsi $\operatorname{Br} X / \operatorname{Br} k$ est d'ordre $p$, engendré par la classe $\pi^{*}(K / k, Q(x))$. Le calcul de résidu a utilisé le fait que ni $Q$ ni $R$ n'ont de zéro dans $K$. 


\section{Arithmétique}

Soient $k$ un corps de nombres et $\Omega$ l'ensemble de ses places. Pour $v \in \Omega$, on note $k_{v}$ le complété de $k$ en $v$ et pour $v$ non archimédienne, $\mathbb{F}_{v}$ le corps résiduel en $v$.

Comme mentionné dans l'introduction, le théorème suivant, avec précisément la même borne $d \geq d_{0}$, est un cas particulier d'un résultat de Wittenberg [17, Thm. 4.8], résultat qui est une conséquence de [6. Thm. 4.1]. Comme nous partons ici d'une hypothèse sur les points rationnels et non sur les zéro-cycles, la démonstration que nous proposons est un peu plus simple que celle de [17, Thm. 4.8]. Comme elle est courte, nous l'avons conservée.

Théorème 3.1. - Soit X une k-variété projective, lisse, géométriquement intègre sur un corps de nombres $k$, équipée d'un morphisme projectif et plat $\pi: X \rightarrow \mathbf{P}_{k}^{1}$ à fibre générique géométriquement intègre. Soit $d_{0}$ la somme des degrés sur $k$ des points fermés de $\mathbf{P}_{k}^{1}$ dont la fibre n'est pas lisse.

Supposons :

(i) Pour tout point fermé $M \in \mathbf{P}_{k}^{1}$, de corps résiduel $k(M)$, la fibre $X_{M} / k(M)$ contient une composante irréductible $Z \subset X_{M}$, de multiplicité 1 , telle que la fermeture algébrique de $k(M)$ dans le corps des fonctions de $Z$ est une extension abélienne de $k(M)$.

(ii) Il existe un adèle dans $X\left(\mathbf{A}_{k}\right)$ orthogonal au groupe de Brauer vertical de $X$, c'est-à-dire au sous-groupe de $\operatorname{Br} X$ dont l'image dans $\operatorname{Brk}(X)$ appartient à $\pi^{*} \operatorname{Br} k\left(\mathbf{P}^{1}\right)$.

(iii) Le principe de Hasse vaut pour les fibres lisses de $\pi$ au-dessus de tout point fermé de $\mathbf{P}_{k}^{1}$.

Alors, pour tout entier $d \geq d_{0}$, et tout ouvert de Zariski non vide $V \subset \mathbf{P}_{k}^{1}$, il existe un point fermé $m \in V$ de degré $d$ sur $k$, à fibre $X_{m}$ lisse contenant un $k(m)$-point rationnel.

Démonstration. - On peut supposer que la fibre de $\pi$ au-dessus du point à l'infini de $\mathbf{P}_{k}^{1}$ est lisse. Soit $V \subset \mathbf{A}_{k}^{1}$ le complémentaire de l'ensemble des points fermés $M$ dont la fibre $X_{M} / k(M)$ n'est pas lisse. Soit $U=\pi^{-1}(V)$. La projection $\pi: U \rightarrow V$ est donc lisse. Il suffit de modifier la démonstration de [6, Thm. 4.1]. Nous supposons que le lecteur a le texte [6] sous la main.

À la page 19 de [6], au lieu d'appliquer [5, Thm. 3.2.2] aux zéro-cycles, on applique [5, Thm. 3.2.1] aux points rationnels, c'est-à-dire le lemme formel d'Harari, comme c'est fait dans [6, (1.4)]. C'est-à-dire que l'on commence 
comme dans la démonstration de [6. Thm. 1.1], plus précisément comme au premier paragraphe de [6, p. 7].

Ceci donne des égalités

$$
\sum_{v \in S_{1}} A_{i, j}\left(p_{v}\right)=0 \in \mathbb{Q} / \mathbb{Z}
$$

avec des $p_{v} \in U\left(k_{v}\right)$ et $S_{1}$ un ensemble fini de places contenant l'ensemble $S$ des places "de mauvaise réduction".

En utilisant le théorème des fonctions implicites, pour chaque place $v$ on peut trouver des $p_{v, l} \in U\left(k_{v}\right), l=1, \ldots, d$, d'images respectives $m_{v, l}=$ $\pi\left(p_{v, l}\right)$, tous distincts dans $V\left(k_{v}\right)$, tels que $A_{i, j}\left(p_{v, l}\right)=A_{i, j}\left(p_{v}\right) \in \operatorname{Br} k_{v}$ pour tout $l$.

On reprend alors la démonstration de [6. Thm. 4.1], page 19, à la formule (4.3), en définissant pour chaque $v \in S_{1}$ le zéro-cycle $z_{v}^{2}$ sur $U$

$$
z_{v}^{2}=\sum_{i=1}^{d} p_{v, l} .
$$

On continue alors la démonstration, avec des polynômes $G_{v}(t)$ unitaires de diviseur $\pi\left(z_{v}^{2}\right)$ sur $\mathbf{A}_{k_{v}}^{1}$. Ces polynômes sont de degré $d$ (au lieu de $1+D d s$ avec les notations de [6]). Procédant comme au troisième paragraphe de la page 20 de [6], en utilisant l'astuce de Salberger, comme détaillée au $\S 3$ de [6]), on trouve un polynôme irréductible $G(t) \in k[t]$, de degré $d$, définissant un point fermé $m \in V$ dont la fibre $X_{m} / k(m)$ a des points dans tous les complétés de $k(m)$, donc a un $k(m)$-point rationnel.

Soit $k$ un corps parfait, $\bar{k}$ une clôture séparable de $k$. Pour $N>0$ entier, et toute $k$-variété quasi-projective $U$, on note $\operatorname{Sym}^{N} U$ le quotient de l'action du groupe symétrique $\mathfrak{S}_{N}$ sur le produit $U^{N}$. On a une bijection entre les ensembles suivants :

(i) Les $k$-points de $\operatorname{Sym}^{N} U$.

(ii) Les zéro-cycles effectifs de degré $N$ sur $U$.

(iii) Les zéro-cycles effectifs de degré $N$ sur $U \times_{k} \bar{k}$ qui sont invariants sous l'action de $\operatorname{Gal}(\bar{k} / k)$.

La bijection entre (ii) et (iii) est claire. Pour tout $k$-point de $\operatorname{Sym}^{N} U$ on choisit un point $\left(P_{1}, \ldots, P_{N}\right) \in U^{N}(\bar{k})$. Le zéro-cycle $P_{1}+\cdots+P_{N}$ est invariant sous $\mathrm{Gal}(\bar{k} / k)$. Inversement si $P_{1}+\cdots+P_{N}$ est un zéro-cycle effectif de degré $N$ sur $U \times_{k} \bar{k}$ invariant sous l'action de $\operatorname{Gal}(\bar{k} / k)$, alors l'image de $\left(P_{1}, \ldots, P_{N}\right) \in U^{N}(\bar{k})$ dans $\left(\operatorname{Sym}^{N} U\right)(\bar{k})$ est invariante sous $\mathrm{Gal}(\bar{k} / k)$, donc définit un $k$-point de $\operatorname{Sym}^{N} U$. 
Corollaire 3.2. - Soit $k$ un corps de nombres. Soit $K / k$ une extension finie cyclique de corps, de degré $p$. Soit $P(x)=Q(x) R(x)$ un polynôme séparable de degré $2 p$ produit de deux polynômes irréductibles de degré $p$. Soit $U=$ $U(K / k, P)$ la k-variété affine, lisse, intègre définie par l'équation

$$
\operatorname{Norm}_{K / k}(\Xi)=P(x) \neq 0 .
$$

Soit $X$ une $k$-variété projective et lisse, géométriquement intègre, contenant $U$ comme ouvert dense. S'il existe un élément $\left\{M_{v}\right\}$ du produit $\prod_{v \in \Omega} U\left(k_{v}\right)$ orthogonal à $(K / k, Q(x)) \in \operatorname{Br} X$, alors il existe une extension de corps $L / k$ de degré $2 p+1$ avec $U(L) \neq \emptyset$. En particulier les $k$-variétés $\operatorname{Sym}^{2 p+1} U$ et Sym $^{2 p+1} X$ possèdent un k-point.

Démonstration. - D'après la proposition 2.2, il existe un tel modèle $X$. Les propriétés d'invariance birationnelle du groupe de Brauer montrent que l'énoncé ne dépend pas du choix du modèle. D'après la proposition 2.2 les classes $(K / k, Q(x))_{k(X)}$ et $(K / k, R(x))_{k(X)}$ sont chacune dans $\operatorname{Br} X$, leur somme dans $\operatorname{Br} k(X)$ est dans l'image de $\operatorname{Br} k$, et ce sont des générateurs de $\operatorname{Br} X$. Les hypothèses du théorème 3.1 sont clairement satisfaites, avec $d_{0}=2 p$. On choisit ici $d=2 p+1$.

Remarque 3.3. - Dans le cadre du corollaire, les seuls $A_{i, j}$ intervenant dans la preuve du théorème 3.1 sont $(K / k, Q(x))_{k(X)}$ et $(K / k, R(x))_{k(X)}$, et ces éléments de $\operatorname{Br} k(X)$ sont ici dans $\operatorname{Br} X$. On n'a donc pas besoin ici d'invoquer le lemme formel d'Harari, l'hypothèse donne les égalités

$$
\sum_{v \in S_{1}} A_{i, j}\left(M_{v}\right)=0 \in \mathbb{Q} / \mathbb{Z},
$$

avec $M_{v}$ comme dans l'énoncé du corollaire, et avec $S_{1}$ égal à l'ensemble fini $S$ évident des places de mauvaise réduction.

Si l'on se limite à $p$ premier impair, ce qui suffirait ici, on peut aussi éviter les difficultés spécifiques aux places réelles (voir à ce sujet [5, p. 82, 1. 6/8] et [6. p. 17, 1. 9/11]).

Proposition 3.4. - Soient $p$ un nombre premier et $k$ un corps de nombres contenant une racine primitive p-ième de l'unité $\zeta$. Il existe $d \in k^{\times}, d \notin k^{\times p}$ et un polynôme séparable $P(x)=Q(x) R(x) \in k[x]$ avec $Q(x)$ et $R(x)$ irréductibles de degré $p$, sans zéro dans $K=k\left(d^{1 / p}\right)$, tels que toute $k$-variété $X$ projective, lisse, géométriquement connexe $k$-birationnelle à la $k$-variété $U=U(K / k, P)$ d'équation affine

$$
\operatorname{Norm}_{K / k}(\Xi)=P(x) \neq 0
$$


satisfasse :

(i) X possède des points rationnels dans tous les complétés $k_{v}$ de $k$.

(ii) $X$ ne possède pas de zéro-cycle de degré 1 . Plus précisément, l'algèbre $A=(K / k, Q(x))=(d, Q(x))_{\zeta} \in \operatorname{Br} k(X)$ appartient à $\operatorname{Br} X$ et, pour toute famille $\left\{z_{v}\right\}, v \in \Omega$, de zéro-cycles de degré 1 sur $X$, on a

$$
\sum_{v \in \Omega} A\left(z_{v}\right) \neq 0 \in \mathbb{Q} / \mathbb{Z}
$$

(iii) On a $\left(\operatorname{Sym}^{2 p+1} U\right)(k)=\emptyset$ et $\left(\operatorname{Sym}^{2 p+1} X\right)(k)=\emptyset$.

Démonstration. - Si une $k$-variété $X$ satisfait (i) et (ii), toute autre $k$-variété projective, lisse, géométriquement connexe $k$-birationnelle à $X$ satisfait (i) et (ii).

L'énoncé (iii) est une conséquence immédiate de l'énoncé (ii). En effet $U$ possède de façon évidente un point dans une extension de degré $p$.

Poonen [11, §5, Prop. 5.1] (cas $p=2$ ) puis de façon semblable VárillyAlvarado et Viray [15, Prop. 4.1] (cas $p$ impair) construisent (au moins biratonnellement) une telle variété $X$ avec des points rationnels dans tous les $k_{v}$ mais telle qu'il y ait obstruction de Brauer-Manin à l'existence d'un point rationnel. Nous allons montrer que leur exemple satisfait aussi l'énoncé analogue pour les zéro-cycles de degré 1, à savoir l'énoncé (ii) de la proposition. Ceci ne résulte pas formellement du cas des points rationnels (cf. [5, §10]).

Soit $M \in \mathbb{N}$ un entier tel que toute courbe plane projective lisse (et donc géométriquement intègre) de degré $p$ sur un corps fini $\mathbb{F}$ de cardinal au moins $M$ possède au moins $2 p+1$ points $\mathbb{F}$-rationnels.

En utilisant le théorème de densité de Chebotarev et la théorie du corps de classes, on trouve $a, b, c \in k$ avec les propriétés suivantes :

(1) $b \in O_{k}, b O_{k}$ idéal premier, $b \equiv 1 \bmod (1-\zeta)^{2 p-1} O_{k}, b>>0$ et $\# \mathbb{F}_{b}>M$,

(2) $a \in O_{k}, a O_{k}$ idéal premier distinct de $b O_{k}, a \equiv 1 \bmod (1-\zeta)^{2 p-1} O_{k}$, $a \notin k_{b}^{\times p}, a>>0$ et $\# \mathbb{F}_{a}>M$.

(3) $c \in O_{k}, b \mid a c+1$.

Soient $d=a b$ et $K$ le corps $k\left(d^{1 / p}\right)$. Soient $Q(x)=x^{p}+c$ et $R(x)=$ $a x^{p}+a c+1$.

Soit $X$ une $k$-variété projective, lisse, géométriquement connexe et $k$ birationnelle à la $k$-variété $U$ d'équation

$$
\operatorname{Norm}_{K / k}(\Xi)=Q(x) R(x) \neq 0 \text {. }
$$


Suivant [11, Lemma 5.3], montrons que l'on a $U\left(k_{v}\right) \neq \emptyset$ pour toute place $v$.

Comme on a $d>>0$, on a $U\left(k_{v}\right) \neq \emptyset$ pour toute place archimédienne.

Soit $v$ une place finie de $k$ différente de $v=v_{a}, v=v_{b}$ et telle que $v(p)=0$. L'extension $k\left(d^{1 / p}\right) / k$ est non ramifiée en $v$. Pour tout $x \in k_{v}$ avec $v(x)<0$, on a $v(P(x))=2 p$, on a donc des points dans $U\left(k_{v}\right)$ avec un tel $x$.

Soit $v$ une place avec $v(p)>0$. L'entier $d$ satisfait $d=a b \equiv 1 \bmod (1-$ $\zeta)^{2 p-1} O_{k}$. Soit $h(x)=x^{p}-d$. Les éléments $p$ et $(1-\zeta)^{p-1}$ diffèrent par une unité dans $\mathbb{Q}(\zeta)$. On en déduit $v(h(1))>2 v\left(h^{\prime}(1)\right)$, où $h^{\prime}(x)=p x^{p-1}$ est le polynôme dérivé de $h(x)$. Le lemme de Hensel assure alors l'existence d'une solution (entière et congrue à 1 modulo $v$ ) de $x^{p}-d=0$ dans $k_{v}$. On a donc $U\left(k_{v}\right) \neq \emptyset$.

Soit $v=v_{b}$. Alors $a$ et $c$ sont des unités dans $k_{v}$. Les hypothèses assurent que la courbe affine lisse $z^{p}=a\left(x^{p}+c\right) \neq 0$ sur le corps $\mathbb{F}_{v}$ possède un point $\mathbb{F}_{v}$-rationnel. Il en est donc de même de la courbe $z^{p}=\left(x^{p}+c\right)\left(a x^{p}+a c+1\right) \neq$ 0 puisque $v_{b}(a c+1)>0$. Par le lemme de Hensel, un tel point se relève en un point de $z^{p}=\left(x^{p}+c\right)\left(a\left(x^{p}+c\right)+1\right) \neq 0$ dans $k_{v}$. Ainsi $U\left(k_{v}\right) \neq \emptyset$.

Soit $v=v_{a}$. Sur le corps $\mathbb{F}_{v}$, on trouve une solution de $z^{p}=x^{p}+c \neq 0$, donc une solution de $z^{p}=\left(x^{p}+c\right)\left(a\left(x^{p}+c\right)+1\right) \neq 0$. Une telle solution se relève en une solution de $z^{p}=\left(x^{p}+c\right)\left(a\left(x^{p}+c\right)+1\right) \neq 0$ dans $k_{v}$. On a donc $U\left(k_{v}\right) \neq \emptyset$.

D'après la proposition 2.2, l'algèbre cyclique $A=(K / k, Q(x))=$ $(d, Q(x))_{\zeta}$ appartient à $\operatorname{Br}(X)$.

Soit $v$ une place de $k$ et soit $L / k_{v}$ une extension finie de corps, et $w$ la valuation sur $L$. L'application $e v_{A}: X(L) \rightarrow \operatorname{Br} L \subset \mathbb{Q} / \mathbb{Z}$ obtenue par évaluation de $A$ est continue. Par le théorème des fonctions implicites, son image est la même que l'image de l'évaluation de $A$ sur $U(L)$ :

$$
e v_{A}: U(L) \rightarrow \operatorname{Br} L \subset \mathbb{Q} / \mathbb{Z}, P \mapsto A(P)
$$

Si $d$ est une puissance $p$-ième dans $L$, l'image de $e v_{A}$ est clairement nulle. C'est le cas pour $v$ place complexe et aussi pour $v$ une place réelle, puisque l'on a $a>>0$ et $b>>0$, et donc $d>>0$. C'est aussi le cas si $v(p)>0$.

Soit $v$ une place non archimédienne de $k$ distincte de $v_{a}$ et de $v_{b}$, avec $v(p)=0$. Si $d$ est une puissance $p$-ième dans $L$, l'image de $e v_{A}$ est nulle. Supposons que $d$ ne soit pas une puissance $p$-ième dans $L$. L'extension de corps locaux $K L / L$ est non ramifiée, les normes sont exactement les éléments de valuation divisible par $p$. Soit $(\Xi, x) \in U(L)$. De l'équation de $U$ on déduit $w(Q(x))+w(R(x)) \equiv 0 \bmod p$. Si $w(x)<0$, alors $w(Q(x))=w\left(x^{p}+c\right)=$ 
$p w(x) \equiv 0 \bmod p$. Supposons $w(x) \geq 0$. On a $\left(a x^{p}+a c+1\right)-a\left(x^{p}+c\right)=1$. On a donc soit $0=w\left(a x^{p}+a c+1\right)$ soit $0=w(a)+w\left(x^{p}+c\right)=w\left(x^{p}+c\right)$. Donc $w(Q(x))=0$ ou $w(R(x))=0$. Comme on a $w(Q(x))+w(R(x)) \equiv$ $0 \bmod p$, on en déduit $w(Q(x)) \equiv 0 \bmod p$. Dans tous les cas, on a donc $w(Q(x)) \equiv 0 \bmod p$, et ceci implique $(d, Q(x))_{\zeta}=0$. On voit donc que pour toute place $v$ non archimédienne de $k$ distincte de $v_{a}$ et de $v_{b}$, et toute extension finie $L / k_{v}$, l'application $e v_{A}: U(L) \rightarrow \operatorname{Br} L \subset \mathbb{Q} / \mathbb{Z}$ a son image nulle.

Soit $v=v_{a}$. Si $w(x)<0$ alors $Q(x)=x^{p}+c$ est une puissance $p$-ième dans $L$, et donc $(d, Q(x))_{\zeta}=0$. Supposons $w(x) \geq 0$. Alors $a x^{p}+a c+1 \equiv$ $1 \bmod a$, ce qui implique que $R(x)=a x^{p}+a c+1$ est une puissance $p$-ième dans $L$, et donc $(d, Q(x))_{\zeta}=(d, R(x))_{\zeta}=0$.

On voit donc que pour toute place $v \neq v_{b}$ et tout zéro-cycle $z_{v}$ sur $X_{k_{v}}$, on a $A\left(z_{v}\right)=0$.

Soit enfin $v=v_{b}$. L'équation de $U$ donne

$$
(d, Q(x))_{\zeta}+\left(d, R(x)_{\zeta}\right)=0 \in \mathbb{Z} / p \subset \mathbb{Q} / \mathbb{Z}=\operatorname{Br} L
$$

Supposons $w(x) \neq 0$. On a $w(a c+1)>0$. Ainsi $R(x)=a x^{p}+a c+1$ est le produit de $a$ et d'une puissance $p$-ième dans $L$. Donc $(d, R(x))_{\zeta}=(a b, a)_{\zeta}$, et donc $(d, Q(x))_{\zeta}=-(a b, a)_{\zeta} \in \mathbb{Z} / p$. Supposons $w(x)>0$. De $w(a c+1)>0$ on déduit que $c$ est une unité dans $L$ et que $-a c$ est une puissance $p$-ième dans $L$. Alors $Q(x)=x^{p}+c$ est le produit de $-a^{-1}$ et d'une puissance $p$ ième dans $L$, donc $(d, Q(x))_{\zeta}=\left(a b,-a^{-1}\right)_{\zeta}=-(a b,-a)_{\zeta}$. Si $p$ est impair, -1 est une puissance $p$-ième, donc $-(a b, a)_{\zeta}=-(a b,-a)_{\zeta}$. Si $p=2$, on a encore cette égalité, car $(a b,-1)_{v_{b}}=0$ [11, Lemma 5.2]. Ceci est établi en utilisant $(a,-1)_{v_{b}}=0$ (facile) et $(b,-1)_{v_{b}}=0$ (obtenu via la formule du produit). En conclusion, pour $v=v_{b}$, toute extension finie $L$ de $k_{v_{b}}$ et tout point $P \in U(L)$, on a $A(P)=-(a b,-a)_{\zeta}=\left(b^{-1},-a\right)_{\zeta}=\left(b^{-1}, a\right)_{\zeta} \in \operatorname{Br} L$.

Pour toute extension finie $E / F$ de corps, le composé $\mathrm{Br} F \rightarrow \operatorname{Br} E \rightarrow \operatorname{Br} F$ de la restriction et de la corestriction est la multiplication par le degré $[E: F]$. Pour $v=v_{b}$ et $z_{v_{b}}=\sum_{i} n_{i} P_{i}$ un zéro-cycle sur $X_{k_{v}}$, la valeur prise par $A$ sur ce zéro-cycle est donc

$$
\sum n_{i}\left[k\left(P_{i}\right): k\right]\left(b^{-1}, a\right)_{\zeta} \in \operatorname{Br} k_{v}
$$

soit encore $\operatorname{deg}\left(z_{v_{b}}\right)\left(b^{-1}, a\right)_{\zeta} \in \operatorname{Br} k_{v_{b}}$. Si le zéro-cycle $z_{v_{b}}$ est de degré 1 , la valeur prise est $\left[\left(b^{-1}, a\right)_{\zeta}\right]_{v_{b}} \in \operatorname{Br} k_{v_{b}} \subset \mathbb{Z} / p$, et cette classe est non nulle, car l'unité $a$ n'est pas une puissance $p$-ième dans le corps résiduel de $k_{v_{b}}$. 
On voit donc que pour toute famille $\left\{z_{v}\right\}, v \in \Omega$, de zéro-cycles de degré 1 sur $X$, on a

$$
\sum_{v \in \Omega} A\left(z_{v}\right)=\left[\left(b^{-1}, a\right)_{\zeta}\right]_{v_{b}} \neq 0 \in \mathbb{Q} / \mathbb{Z}
$$

\section{Le théorème}

Soient $p$ un nombre premier et $k$ un corps de nombres contenant une racine primitive $p$-ième $\zeta$ de 1 , qu' on fixe, déterminant ainsi un isomorphisme $\mathbb{Z} / p \stackrel{\widetilde{\sim}}{\rightarrow} \mu_{p}$ sur $k$. Soient $a, b, c, d \in k$ et $Q(x), R(x), P(x)=Q(x) R(x)$ comme dans la proposition 3.4 et sa démonstration.

Soient $A=k[u, 1 / u]$ et $B=k[u, 1 / u][v] /\left(v^{p}-d u\right)$, avec le plongement évident $A \hookrightarrow B$, qui fait de Spec $B$ un $A$-schéma fini étale, plus précisément un $\mathbb{Z} / p$-torseur.

Soit $\mathbb{U}$ le $R$-schéma lisse défini par

$$
\operatorname{Norm}_{B / A}(\Xi)=P(x) \neq 0,
$$

où $\Xi=\sum_{i=0}^{p-1} v^{i} x_{i}$. L'espace total $\mathbb{U}$ est affine, car donné par le système

$$
\operatorname{Norm}_{B / A}(\Xi)=P(x), P(x) y-1=0 .
$$

La fibre de $\mathbb{U} / R$ en un point $u \in \operatorname{Spec} R$ est notée $\mathbb{U}_{u}$. Pour $u$ un $k$-point, $\mathbb{U}_{u}=U\left(k\left((d u)^{1 / p}\right) / k, P(x)\right)$.

En utilisant le théorème d'Hironaka, on construit un $A$-schéma intègre $\mathbb{X}$, projectif et lisse sur $A$, contenant le $A$-schéma $\mathbb{U}$ comme ouvert, tel que pour tout $k$-homomorphisme $A \rightarrow k$, i.e. pour tout choix de $u \in k^{\times}$, le $A$-schéma $\mathbb{X}$ se spécialise en une $k$-variété projective, lisse, géométriquement intègre $\mathbb{X}_{u}=X_{k\left((d u)^{1 / p}\right) / k}$ contenant $U\left(k\left((d u)^{1 / p}\right) / k, P(x)\right)$ comme ouvert dense.

Lemme 4.1. - Soit C la k-courbe lisse définie par l'équation

$$
z^{p}=Q(x) R(x) \neq 0 .
$$

Il existe un ensemble fini $S$ de places de $k$, contenant les places archimédiennes, tel que, pour toute place $v \notin S$,

(i) On a $C\left(k_{v}\right) \neq \emptyset$.

(ii) Pour tout $u \in k_{v}^{\times}$de valuation $v$-adique $v(u)$ non nulle modulo $p$, l'algèbre $(u, Q(x))_{\zeta}$, quand évaluée sur $C\left(k_{v}\right)$, prend toutes les valeurs dans $\mathbb{Z} / p \subset \operatorname{Br} k_{v}$. 
Démonstration. - Soit $v$ une place finie telle que $Q(x) R(x)$ ait ses coefficients dans l'anneau $O_{v}$ des entiers de $k_{v}$, son coefficient dominant une unité dans $O_{v}$, et que $p$ soit inversible dans $O_{v}$. Soit $\mathbb{F}_{v}$ le corps résiduel en $v$. La $\mathbb{F}_{v}$-courbe $C_{\gamma}$ définie par

$$
z_{1}^{p}=\gamma Q(x) \neq 0, z_{2}^{p}=\gamma^{-1} R(x) \neq 0,
$$

est lisse et géométriquement intègre. Son modèle projectif évident dans $\mathbf{P}^{3}$ est une courbe intersection complète lisse $D_{\gamma}$ de deux surfaces de degré $p$, le genre de $D_{\gamma}$ est donc $p^{3}-2 p^{2}+1$. Sur une clôture algébrique du corps de base, on vérifie immédiatement que le complémentaire de $C_{\gamma}$ dans $D_{\gamma}$ est formé de $3 p^{2}$ points.

Si le cardinal de $\mathbb{F}_{v}$ est plus grand qu'une constante dépendant seulement de l'entier $p$, par les estimations de Weil pour les courbes, pour tout $\gamma \in \mathbb{F}_{v}^{\times}$, la $\mathbb{F}_{v}$-courbe $C_{\gamma}$ contient un $\mathbb{F}_{v}$-point. En associant au point de coordonnées $\left(x, z_{1}, z_{2}\right)$ le point de coordonnées $\left(x, z=z_{1} . z_{2}\right)$ on définit un morphisme de $C_{\gamma}$ dans la courbe lisse d'équation $z^{p}=Q(x) R(x) \neq 0$ sur $\mathbb{F}_{v}$. Par le lemme de Hensel, l'image d'un $\mathbb{F}_{v}$-point de $C_{\gamma}$ dans cette courbe lisse est un $\mathbb{F}_{v}$-point qui se relève en un $O_{v}$-point de la courbe $C$. Sur un tel $O_{v}$-point, l'algèbre $(u, Q(x))_{\zeta}$ a pour valeur $\gamma^{v(u)} \in \mathbb{F}_{v}^{\times} / \mathbb{F}_{v}^{\times p} \simeq \mathbb{Z} / p$. Comme $\gamma \in \mathbb{F}_{v}^{\times}$ est arbitraire, ceci établit le lemme.

L'énoncé suivant est la généralisation du théorème 1.3 de [10] (cas $p=2)$. C'est le Théorème 5.2 de [15].

Proposition 4.2. - L'ensemble des $u \in k^{\times}$tels que que l'on ait à la fois $\mathbb{X}_{u}\left(\mathbf{A}_{k}\right) \neq \emptyset$ et $\mathbb{X}_{u}\left(\mathbf{A}_{k}\right)^{\mathrm{Br}}=\emptyset$ forme un nombre fini de classes dans $k^{\times} / k^{\times p}$.

Démonstration. - Il est clair que chacune des deux propriétés considérées ne dépend que de la classe de $u$ dans $k^{\times} / k^{\times p}$. Soient $C$ et $S$ comme dans le lemme 4.1. On suppose de plus que $S$ contient les places finies avec $v(d) \neq 0$, c'est-à-dire $v_{a}$ et $v_{b}$. Pour tout $u \in k^{\times}$, la courbe $C$ est contenue dans $\mathbb{X}_{u}$. Soit $u$ tel que $\mathbb{X}_{u}\left(\mathbf{A}_{k}\right) \neq \emptyset$. S'il existe une place $v \notin S$ telle que $v(u)=$ $v(d u) \in \mathbb{Z}$ ne soit pas divisible par $p$, alors, d'après le lemme 4.1, l'algèbre $(d u, Q(x))_{\zeta}$ parcourt sur $C\left(k_{v}\right) \subset \mathbb{X}_{u}\left(k_{v}\right)$ toutes les valeurs dans $\mathbb{Z} / p \subset$ $\operatorname{Br} k_{v}$. D'après la proposition 2.2, la classe de $(d u, Q(x))_{\zeta}$ appartient à $\mathrm{Br} \mathbb{X}_{u}$ et engendre $\operatorname{Br}_{u} / \operatorname{Br} k$. Il ne saurait donc y avoir obstruction de Brauer-Manin à l'existence d'un $k$-point, et encore moins d'un zéro-cycle de degré 1 sur $\mathbb{X}_{u}$. On voit donc que les $u \in k^{\times}$tels que $\mathbb{X}_{u}\left(\mathbf{A}_{k}\right) \neq \emptyset$ et qu'on ait obstruction de Brauer-Manin à l'existence d'un zéro-cycle de degré 1 ont leur image dans le 
noyau de l'application

$$
\operatorname{div}: k^{\times} / k^{\times p} \rightarrow \oplus_{v \notin S} \mathbb{Z} / p
$$

et ce noyau est fini (finitude du nombre de classes et théorème des unités de Dirichlet).

Nous pouvons maintenant donner la démonstration du théorème principal.

Théorème 4.3. - Soit $k$ un corps de nombres. Soit $r>0$ un entier. Le complément de $k^{\times r}$ dans $k^{\times}$est un ensemble diophantien : il existe une $k$-variété $Z$ et un k-morphisme $f: Z \rightarrow \mathbf{A}_{k}^{1}$ tel que $f(Z(k))$ soit le complémentaire des puissances $r$-ièmes dans $k$.

Démonstration. - Une réduction connue [10, Cor. 1.2], [15, Lemma 5.3 \& Proof of Cor. 1.2, p. 133] montre qu'il suffit d'établir le résultat lorsque $r$ est un nombre premier $p$, et que de plus $k$ contient les racines primitives $p$-ièmes de l'unité. On fixe une telle racine primitive $p$-ième $\zeta$ dans $k$.

Notons $H_{v}=k^{\times} \cap k_{v}^{\times p}$ et $N_{v}$ le complémentaire de $H_{v}$ dans $k^{\times}$. Ce sont des ensembles diophantiens (cf. [10, Thm 1.5]) stables par multiplication par $k^{\times p}$.

Soit $D_{1}$ l'ensemble des $u \in k^{\times}$tels que $\operatorname{Sym}^{2 p+1} \mathbb{U}_{u}(k) \neq \emptyset$. C'est un ensemble stable par multiplication par $k^{\times p}$. C'est un ensemble diophantien, car c'est l'image des points $k$-rationnels de la $k$-variété $\operatorname{Sym}_{A}^{2 p+1} \mathbb{U}$ par la projection naturelle $\operatorname{Sym}_{A}^{2 p+1} \mathbb{U} \rightarrow \operatorname{Spec} A=\mathbb{G}_{m, k} \subset \mathbf{A}_{k}^{1}$. On a noté ici $\operatorname{Sym}_{A}^{2 p+1} \mathbb{U}$ le quotient du produit fibré de $N$ exemplaires de $\mathbb{U}$ au-dessus de $\operatorname{Spec} A$ par l'action de $\mathfrak{S}_{N}$.

Soit $D_{2}$ la réunion de $D_{1}$ et des $N_{v}$ pour $v \in S$, avec $S$ comme au lemme 4.1. C'est un ensemble diophantien dans $k^{\times} \subset k$, stable par multiplication $\operatorname{par} k^{\times p}$.

Considérons le complément $E$ de $D_{2}$ dans $k^{\times}$. C'est un ensemble stable par multiplication par $k^{\times p}$. Pour tout $u \in E$, on a $\mathbb{X}_{u}\left(\mathbf{A}_{k}\right) \neq \emptyset$. D'après le corollaire 3.2, l'ensemble $E$ est contenu dans l'ensemble $C$ des $u \in k^{\times}$ tels que de plus $\mathbb{X}_{u}\left(\mathbf{A}_{k}\right)^{\mathrm{Br}}=\emptyset$. D'après la proposition 4.2, l'ensemble $C$ est union d'un nombre fini d'orbites de $k^{\times p}$ dans $k^{\times}$. Il en est donc de même de $E$. D'après la proposition 3.4 l'orbite de 1 , soit $k^{\times p}$, n'est pas dans $D_{2}$, et elle est dans $E$.

Le complément $D_{3}$ de $k^{\times p}$ dans $E$ est une union finie d'orbites de $k^{\times p}$ dans $k^{\times}$, chacune clairement un ensemble diophantien, donc $D_{3}$ est un ensemble diophantien. La réunion de $D_{2}$ et de $D_{3}$ est un ensemble diophantien $D_{4}$ dont 
le complément dans $k^{\times}$est précisément $k^{\times p}$. Ainsi le complément de $k^{\times p}$ dans $k^{\times}$est l'ensemble diophantien $D_{4}$, ce qui établit le théorème 4.3 .

Remarque 4.4. - Dans la démonstration, on peut remplacer l'ensemble diophantien $D_{1}$ par l'ensemble diophantien $D_{1}^{\prime}$ formé des $u \in k^{\times}$tels que $\operatorname{Sym}^{2 p+1} \mathbb{X}_{u}(k) \neq \emptyset$. L'ensemble $D_{1}^{\prime}$ est stable par multiplication par $k^{\times p}$. Il contient $D_{1}$. On définit $D_{2}^{\prime}$ comme la réunion de $D_{1}^{\prime}$ et des $N_{v}$ pour $v \in S$, puis $E^{\prime} \subset E \subset C$ comme le complément de $D_{2}^{\prime}$ dans $k^{\times}$.

\section{Une variante de la démonstration}

Dans la démonstration du théorème 4.3, un ingrédient clé est le corollaire 3.2 du théorème 3.1

En combinant un résultat non publié de Salberger (1985), décrit dans l'appendice (paragraphe 6 ci-après), on peut utiliser directement les théorèmes principaux de [5] ou [6] sur les zéro-cycles pour donner une variante de la démonstration du théorème 4.3, variante qui évite le corollaire $3.2 \mathrm{du}$ théorème 3.1, et donc aussi ce dernier théorème.

Le corollaire 3.2 du théorème 3.1 établit le résultat suivant :

Soit $u \in k^{\times}$. Si l'on a $\mathbb{X}_{u}\left(\mathbf{A}_{k}\right)^{\mathrm{Br}} \neq \emptyset$, alors $\operatorname{Sym}^{2 p+1} \mathbb{X}_{u}(k) \neq \emptyset$.

Voici comment établir directement un substitut de ce résultat. D'après [5, Thm. 5.1] ou [6, Thm. 4.1], l'hypothèse $\mathbb{X}_{u}\left(\mathbf{A}_{k}\right)^{\mathrm{Br}} \neq \emptyset$ implique l'existence d'un zéro-cycle de degré 1 sur la $k$-variété $\mathbb{X}_{u}$. Le théorème 6.5 ci-dessous, avec $s=2 p$, donne alors l'existence d'un zéro-cycle effectif de degré $(p-1)^{2}$ sur $\mathbb{X}_{u}$. Ainsi $\mathbb{X}_{u}\left(\mathbf{A}_{k}\right)^{\mathrm{Br}} \neq \emptyset$ implique que $u \in k^{\times}$est dans l'image des points $k$-rationnels de $\operatorname{Sym}_{A}^{N} \mathbb{X}$ via la projection $\operatorname{Sym}_{A}^{p^{2}-2 p+1} \mathbb{X} \rightarrow \mathbb{G}_{m}$. On procède alors exactement comme dans la démonstration du théorème 4.3, en y remplaçant la projection $\operatorname{Sym}_{A}^{p+1} \mathbb{U} \rightarrow \mathbb{G}_{m}$ par $\operatorname{Sym}_{A}^{p^{2}-2 p+1} \mathbb{X} \rightarrow \mathbb{G}_{m}$.

\section{Appendice : Résultats d'effectivité pour les zéro-cycles sur les familles de variétés de Severi-Brauer}

Soit $p$ un nombre premier, $k$ un corps contenant une racine primitive $p$ ième de 1 et $P(x) \in k[x]$ un polynôme séparable de degré $2 p$. Soit $K / k$ une extension cyclique de corps, de groupe $G=\langle\sigma\rangle=\mathbb{Z} / p$.

Soit $R_{1}$ la $k[x]$-algèbre

$$
R_{1}=\oplus_{i=0}^{p-1} K[x] \xi^{i},
$$


avec $x$ central et les relations $\xi^{p}=P(x)$ et pour $\lambda \in K, \xi . \lambda=\sigma(\lambda) . \xi$. Alors $R_{1}$ est un ordre maximal, et donc en particulier héréditaire, dans l'algèbre simple centrale $(K / k, P(x))$ sur sur le corps $k(x)$.

Notons $P^{\prime}(y)=y^{2 p} P(1 / y)$. Soit $R_{2}$ la $k[y]$-algèbre

$$
R_{1}=\oplus_{i=0}^{p-1} K[y] \eta^{i}
$$

avec $y$ central et les relations $\eta^{p}=P^{\prime}(y)$ et, pour $\lambda \in K, \eta \cdot \lambda=\sigma(\lambda)$. $\eta$. Alors $R_{2}$ est un ordre héréditaire sur $k[y]$.

Les identifications $y=1 / x$ et $y^{2} \xi=\eta$ permettent, sur $k\left[x, x^{-1}\right]$ et $k\left[y, y^{-1}\right]$, de recoller les deux ordres héréditaires en un faisceau $\tilde{R}$ de tels ordres sur la droite projective $\mathbf{P}_{k}^{1}$.

D'après $\mathbf{M}$. Artin [1] , le foncteur $F$ sur la catégorie des $\mathbf{P}_{k}^{1}$-schémas dont les points $F(S)$ sur un $\mathbf{P}_{k}^{1}$-schéma affine $S$ sont les idéaux à gauche du faisceau d'algèbres $\tilde{R}_{S}$ qui sont localement libres de rang $p$ comme faisceaux de $O_{S}$-modules, et qui sont localement facteurs directs comme $O_{S}$-modules de $\tilde{R}_{S}$, est représentable par un $\mathbf{P}_{k}^{1}$-schéma projectif $X^{\prime}$.

Au-dessus de l'ouvert de $\mathbf{P}_{k}^{1}$ où l'algèbre $\tilde{R}$ est d'Azumaya, ce schéma $X^{\prime}$ se restreint en un schéma de Severi-Brauer relatif. Toujours d'après M. Artin [1, Thm. (1.4)], la fibre générique de ce $\mathbf{P}_{k}^{1}$-schéma $X^{\prime}$ est la variété de SeveriBrauer sur le corps $k\left(\mathbf{P}^{1}\right)=k(x)$ associée à la $k(x)$-algèbre simple centrale $(K / k, P(x))$.

Toujours d'après M. Artin, la composante connexe du schéma $X^{\prime}$ contenant la fibre générique est un $k$-schéma régulier $X=X(K / k, P)$, donc lisse sur $k$ si car $(k)=0$. Les fibres de $\pi: X \rightarrow \mathbf{P}_{k}^{1}$ sont géométriquement connexes.

M. Artin ([1] , voir aussi [7]), donne une description précise des fibres non lisses du morphisme projectif $X \rightarrow \mathbf{P}_{k}^{1}$, description dont nous n'aurons pas besoin ici.

Le théorème suivant est annoncé et partiellement démontré par P. Salberger dans sa thèse [12].

Théorème 6.1. - [12, Cor. 5.9] Soit $k$ un corps de caractéristique nulle. Soit $K=k\left(\mathbf{P}^{1}\right)$. Soit $A$ une algèbre à division sur $K$ d'indice premier $p$. Soit $\tilde{R}$ un faisceau d'ordres maximaux de $A$ sur $\mathbf{P}_{k}^{1}$. Soit $\pi: X \rightarrow \mathbf{P}_{k}^{1}$ le schéma comme ci-dessus. Soit s la somme des degrés sur $k$ des points fermés de $\mathbf{P}_{k}^{1}$

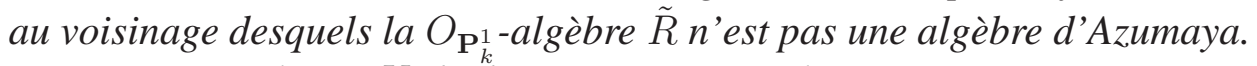

Tout zéro-cycle sur $X$ de degré au moins égal à

$$
N_{0}=(1 / 2)(p-1)(s-2(p+1) / p)
$$

est rationnellement équivalent sur $X$ à un zéro-cycle effectif. 
Ce théorème généralise un résultat sur les surfaces fibrées en coniques audessus de la droite projective [2]. Salberger donne un énoncé plus général, au-dessus d'une courbe de genre quelconque. Sa démonstration utilise une version de l'inégalité de Riemann pour les idéaux des ordres maximaux sur une courbe due à Witt [16], et reprise en particulier dans [14].

Corollaire 6.2. - Avec les notations et hypothèses ci-dessus, soit $N=$ $N(p, s) \geq 1$ le plus petit entier congru à 1 modulo $p$ et au moins égal à $(1 / 2)(p-1)(s-2(p+1) / p)$. Les conditions suivantes sont équivalentes :

(a) X possède un zéro-cycle de degré 1 .

(b) X possède un zéro-cycle effectif de degré $N$.

(c) Le produit symétrique $\mathrm{Sym}^{N} X$ possède un k-point.

Donnons un énoncé analogue pour toute $k$ variété projective et lisse $k$ birationnelle à $X$.

Le lemme suivant est une extension aux zéro-cycles du lemme de LangNishimura [3, Lemme 3.1.1].

Lemme 6.3. - Soit $k$ un corps de caractéristique zéro. Soit $X$ une $k$ variété lisse intègre et $Y$ une $k$-variété propre. S'il existe une application $k$-rationnelle $f$ de $X$ vers $Y$, et si $X$ possède un zéro-cycle effectif de degré $N$, alors $Y$ possède un zéro-cycle effectif de degré $N$.

Démonstration. - Soit $z$ un tel zéro-cycle. On éclate le support du zérocycle. Ceci donne une $k$-variété lisse $X^{\prime}$ et une application $k$-rationnelle $f^{\prime}$ de $X^{\prime}$ vers $Y$, qui est définie sur le complémentaire d'un fermé de codimension 2 de $X^{\prime}$. La fibre au-dessus d'un point fermé $P$ dans le support de $z$ est un espace projectif $\mathbf{P}_{k(P)}^{d-1}$. Il existe donc un zéro-cycle effectif $z^{\prime}$ de degré $N$ dans l'ouvert de définition de $f^{\prime}$. L'image de ce zéro-cycle par l'application $f_{*}^{\prime}$ est un zéro-cycle de degré $N$ dans $Y$.

Lemme 6.4. - Soit $k$ un corps de caractéristique zéro. Soient $X$ et $Y$ deux $k$-variétés propres et lisses, géométriquement connexes, $k$-birationnelles entre elles. Si pour un entier $N>0$ tout zéro-cycle sur $Y$ de degré $N$ est rationnellement équivalent sur $Y$ à un zéro-cycle effectif, et si $X$ possède un zéro-cycle de degré $N$, alors $X$ possède un zéro-cycle effectif de degré $N$.

Démonstration. - Il existe des ouverts non vides $U \subset Y$ et $V \subset X$ qui sont $k$-isomorphes. Soit $z$ un zéro-cycle sur $X$ de degré $N$. Par un lemme de déplacement facile, ce zéro-cycle est rationnellement équivalent sur $X$ à un zéro-cycle de degré $N$ à support dans $V$. On considère le zéro-cycle image 
dans $U$. Il est de degré $N$. Sur $Y$, il est rationnellement équivalent à un zérocycle effectif de degré $N$. Le lemme 6.3 établit l'existence d'un zéro-cycle de degré $N$ sur $X$.

Nous pouvons maintenant énoncer le corollaire suivant du résultat de Salberger.

Théorème 6.5. - Soit $k$ un corps de caractéristique zéro. Soit $X \rightarrow \mathbf{P}_{k}^{1}$ un $k$-morphisme de $k$-variétés projectives et lisses géométriquement intègres dont la fibre générique est k-birationnelle à une variété de Severi-Brauer d'indice premier $p$, d'algèbre associée $A / k\left(\mathbf{P}^{1}\right)$. Soit s la somme des degrés sur $k$ des points fermés de $\mathbf{P}_{k}^{1}$ où l'algèbre $A$ a un résidu non trivial. Soit $N=N(p, s) \geq 1$ un entier congru à 1 modulo $p$ et au moins égal à $(1 / 2)(p-$ $1)(s-2(p+1) / p)$. Les conditions suivantes sont équivalentes:

(a) $X$ possède un zéro-cycle de degré 1 .

(b) X possède un zéro-cycle effectif de degré $N$.

(c) Le produit symétrique $\mathrm{Sym}^{N} X$ possède un k-point.

Démonstration. - Soit $A / K=k\left(\mathbf{P}^{1}\right)$ l'algèbre simple centrale associée à la fibre générique de $\pi$. Si $A$ n'est pas une algèbre à division, alors c'est une algèbre de matrices, et la $k$-variété $X$ est $k$-birationnelle à $\mathbf{P}_{k}^{p-1} \times_{k} \mathbf{P}_{k}^{1}$, donc à $\mathbf{P}_{k}^{p}$. Le groupe de Chow $C H_{0}(X)$ des zéro-cycles modulo l'équivalence rationnelle sur $X$ est alors égal à $\mathbb{Z}$, engendré par la classe d'un point $k$ rationnel, et le théorème est clair.

Supposons que $A$ est une algèbre à division. Soit $\pi: Y \rightarrow \mathbf{P}_{k}^{1}$ un modèle donné par le théorème 6.1 .

La considération d'une fibre lisse sur un $k$-point de $\mathbf{P}_{k}^{1}$ montre que $X$ et $Y$ possèdent soit un $k$-point soit un point fermé de degré $p$ sur $k$. Ceci établit en particulier que (b) implique (a). Supposons (a). D'après ce qui précède, il existe un zéro-cycle de degré $N(p, s)$ sur $X$. Le théorème 6.1 et le lemme6.4 donnent alors (b). Les énoncés (b) et (c) sont clairement équivalents.

\section{Références}

[1] M. Artin, Left ideals in maximal orders, in Brauer Groups in Ring Theory and Algebraic Geometry, LNM 917, Springer-Verlag (1982) p. 182-193.

[2] J.-L. Colliot-Thélène et D. Coray, L'équivalence rationnelles sur les points fermés des surfaces rationnelles fibrées en coniques, Compositio math. 39 (1979) $301-322$. 
[3] J.-L. Colliot-Thélène, D. Coray et J.-J. Sansuc, Descente et principe de Hasse pour certaines variétés rationnelles, Journal für die reine und angewandte Mathematik (Crelle) 320 (1980), 150-191.

[4] J.-L. Colliot-Thélène, J.-J. Sansuc et Sir Peter Swinnerton-Dyer, In tersections of two quadrics and Châtelet surfaces, I, II, Journal für die reine und angewandte Mathematik (Crelle) 373 (1987) 37-107; 374 (1987) 72-168.

[5] J.-L. Colliot-Thélène et Sir Peter Swinnerton-Dyer, Hasse principle and weak approximation for pencils of Severi-Brauer and similar varieties, Journal für die reine und angewandte Mathematik (Crelle) 453 (1994) 49-112.

[6] J.-L. Colliot-Thélène, A. N. Skorobogatov et Sir Peter Swinnerton-Dyer, Rational points and zero-cycles on fibred varieties : Schinzel's hypothesis and Salberger's device, Journal für die reine und angewandte Mathematik (Crelle) 495 (1998) 1-28.

[7] E. Frossard, Fibres dégénérées des schémas de Severi-Brauer d'ordres, J. Alg. 198 (1997), 362-387.

[8] P. Gille et T. Szamuely, Central simple algebras and Galois cohomology, Cambridge studies in advanced mathematics 101 (2006).

[9] J. Königsmann, Defining $\mathbb{Z}$ in $\mathbb{Q}$, arxiv.org/abs/1011.3424v2, à paraître dans Annals of Math.

[10] B. Poonen, The set of nonsquares in a number field is Diophantine, Math. Res. Lett. 16 (2009), no. 1, 165-170.

[11] B. Poonen, Existence of rational points on smooth projective varieties, J. Eur. Math. Soc. 11 (2009) 529-543.

[12] P. Salberger, Class groups of orders and Chow groups of their Brauer-Severi schemes, in K-Theory of orders and their Brauer-Severi schemes, Thèse, Chalmers University of Technology, Göteborg, 1985.

[13] P. Salberger, Zero-cycles on rational surfaces over number fields, Invent. math. 91 (1988), no. 3, 505-524.

[14] M. van den Bergh et J. van Geel, Algebraic elements in division algebras over function fields of curves, Israel J. Math. 52 (1985), 33-45.

[15] A. Várilly-Alvarado et B. Viray, Higher dimensional analogues of Châtelet surfaces, Bull. London Math. Soc. 44 (2012), no. 1, 125-135.

[16] E. Witt, Riemann-Rochscher Satz und Z-Funktion im Hyperkomplexen, Math. Ann. 110 (1934) 12-28.

[17] O. Wittenberg, Zéro-cycles sur les fibrations au-dessus d'une courbe de genre quelconque, Duke Math. J. 161 (2012) 2113-2166.

5 janvier 2014 
Jean-Louis Colliot-Thélène, CNRS \& Université Paris Sud, Mathématiques, Bâtiment 425, F-91405 Orsay Cedex, France • E-mail: jlct@math.u-psud.fr

JAN VAN GEEL, Universiteit Gent, Vakgroep Wiskunde, Krijgslaan 281, S22, B-9000 Gent,

België • E-mail: jvg@cage.ugent.be 